\title{
Calidad de vida en la infancia: estudio comparativo entre una zona rural y urbana en el norte de Chile
}

\author{
ALFONSO URZÚA M. ${ }^{1}$, ALEJANDRA CAQUEO-URÍZAR ${ }^{2}$, NELIA ALBORNOZ B. ${ }^{1}$, CRISTINA JARA S. ${ }^{1}$ \\ 1. Universidad Católica del Norte, Antofagasta, Chile. \\ 2. Universidad de Tarapacá, Arica, Chile.
}

\begin{abstract}
Quality of life in children: a comparative study between urban and rural areas in Northern Chile
\end{abstract}

Background: The quality of life in children is associated with their experiences, family and their sociocultural environment. Objective: To identify the significant components in assessing quality of life from the children's perception. Participants and Method: Using a cross-sectional design, the Child Life Quality Scale was applied to a total of 200 children aged 8-12 years, from an urban and a rural area located in northern Chile. Three open questions were presented about experiences of satisfaction, dissatisfaction and desire for change, classifying the answers in six categories. Results: No significant differences in the perception of quality of life in children from urban and rural areas were found, however, the evaluation of the content of the answers allowed us to establish some differences between the two groups. Conclusion: In the urban area, the greatest experiences of satisfaction level were associated with interpersonal relationships, while in the rural area, with leisure and recreational activities.

(Key words: Quality of life, children, rural, urban, Child Life Quality Scale).

Rev Chil Pediatr 2013; 84 (3): 276-284

\section{RESUMEN}

Introducción: La calidad de vida infantil está asociada con las propias vivencias de los menores, la de sus familias, como con variables del entorno sociocultural donde se desarrollan. Objetivo: Identificar los componentes significativos en la evaluación de la calidad de vida desde la percepción infantil. Participantes y Método: Bajo un diseño transversal, se aplicó la Escala de Calidad de Vida Infantil a un total de 200 niños y niñas de 8 a 12 años de una comuna urbana y de una comuna rural ubicadas al Norte de Chile. Se presentaron tres preguntas abiertas sobre experiencias de satisfacción, insatisfacción y deseos de cambio, clasificando las respuestas en 6 dimensiones. Resultados: Muestran la inexistencia de diferencias en la percepción de calidad

Recibido el 29 de septiembre de 2012, devuelto para corregir el 18 de diciembre de 2012, segunda versión 14 de enero de 2013, tercera versión 13 de marzo de 2013, aceptado para publicación el 18 de marzo de 2013.

Este trabajo cumple con los requisitos sobre consentimiento /asentimiento informado, comité de ética, financiamiento, estudios animales y sobre la ausencia de conflictos de intereses según corresponda.

Correspondencia a:

Alfonso Urzúa M.

E-mail: alurzua@ucn.cl 
de vida en niños urbanos y rurales a partir del cuestionario; sin embargo, la evaluación del contenido de las preguntas abiertas permitió establecer algunas diferencias entre ambos grupos. Conclusión: En la comuna urbana, las mayores experiencias de satisfacción se dieron a nivel de las relaciones interpersonales, en tanto en el sector rural, en torno a las actividades de ocio y recreativas.

(Palabras clave: Calidad de vida, niños, rural, urbano, Escala de Calidad de Vida Infantil).

Rev Chil Pediatr 2013; 84 (3): 276-284

\section{Introducción}

La Organización Mundial de la Salud define a la Calidad de Vida (CV) como la percepción que un individuo tiene de su lugar en la existencia, en el contexto de la cultura y del sistema de valores en los que vive y en relación con sus objetivos, sus expectativas, sus normas y sus inquietudes ${ }^{1}$. Operacionalmente, puede ser entendida como el nivel percibido de bienestar derivado de la evaluación que realiza cada persona de elementos objetivos y subjetivos en distintas dimensiones de su vida ${ }^{2}$. Es un concepto amplio que está influido de modo complejo por la salud física del sujeto, su estado psicológico, su nivel de independencia, sus relaciones sociales, así como su relación con los elementos esenciales de su entorno.

Una de las áreas donde la investigación ha tenido un mayor desarrollo es la de la salud, centrándose en la evaluación de la denominada Calidad de Vida Relacionada con la Salud (CVRS), concebida como el nivel de bienestar derivado de la evaluación que la persona realiza de diversos dominios de su vida, considerando el impacto que en éstos tiene su estado de salud $^{3}$. Los estudios en Calidad de Vida Relacionada con la Salud (CVRS) han predominado tanto en población adulta como en población infantil y adolescente, enfocándose en la percepción que tienen los pacientes acerca de los efectos del padecimiento de la enfermedad, además de las consecuencias de los tratamientos en la vida de las personas, dando un especial énfasis al bienestar físico, emocional y social ${ }^{4}$.

Las principales dimensiones de la Calidad de Vida en la infancia consideradas por los profesionales de salud e investigadores son: estatus funcional, funcionamiento psicológico y funcionamiento social. El estatus funcional se refiere a la habilidad del niño para desempe- ñar actividades diarias apropiadas de su edad, y está íntimamente relacionado con la sintomatología física. El funcionamiento psicológico incluye la evaluación del estado afectivo del paciente y finalmente, el funcionamiento social se relaciona con la habilidad del niño para mantener relaciones íntimas con su familia y amigos ${ }^{5}$.

En Chile las investigaciones referidas a la Calidad de Vida infantil y adolescente aún son escasas y realizadas en población fundamentalmente escolarizada urbana ${ }^{6,7-9}$, o bien vinculadas a patologías ${ }^{10,11}$.

Los principales resultados muestran que la Calidad de Vida es superior en los hombres solamente a nivel de la dimensión del bienestar físico. En los dominios que implican relación con el medio en distintos ámbitos, la media es superior en las mujeres. Las mujeres priorizan actividades destinadas a la socialización, en tanto los hombres lo hacen en deportes y actividad física. A nivel físico, en las mujeres los cambios secundarios y especialmente la menstruación son la causa de muchas complicaciones, siendo los problemas de salud más comunes los desórdenes hormonales, lo que puede incidir en una Calidad de Vida más disminuida en esta dimensión.

En relación a la edad de los menores evaluados, es posible que los más pequeños perciban de mejor manera su apariencia física que los y las adolescentes, dado que aún no comienza el período de desarrollo y con esto los problemas generados con el cuerpo por el surgimiento de las características sexuales secundarias.

Desde un punto de vista socio evolutivo, niños y niñas invierten más tiempo en actividades escolares y tienen una mejor relación con sus padres, ya que son más dependientes de ellos. Esto último no ocurre en la adolescencia, debido al proceso de individualización ${ }^{8}$.

Un avance importante en estos estudios ha 
sido el que ha permitido recoger la percepción subjetiva de los niños y adolescentes, aportando información no sólo referida a los niños, sino que desde la propia perspectiva de estos. Esto es relevante, dado que deja atrás la visión adultocentrista, preocupándose de las necesidades de los niños y niñas, inmersos en sus diversos contextos socioculturales ${ }^{12}$, considerándolos como sujetos sociales con capacidad de expresar sus opiniones y decisiones en asuntos que les competen directamente en la familia, la escuela y la sociedad en general ${ }^{13}$.

Se observa que la evaluación que hacen los niños está asociada con sus propias vivencias y la de sus familias, así como con aspectos que tienen que ver con el entorno social en el que viven ${ }^{14}$. No obstante, los estudios requieren generalmente contextualización apropiada según el lugar geográfico, ámbito (educación, servicios sociales y salud), grupos de personas objeto de la evaluación y otros aspectos. En este sentido, es necesario desarrollar diferentes escalas para evaluarlos ${ }^{15}$ considerando que la forma de interactuar de los niños en su medio obedece en gran medida al descernimiento que les permite aprender "de" y "a partir" de los otros miembros de su cultura ${ }^{16}$. Se ha visto que dichas diferencias (junto a otras variables dinámicas como género, raza, etnicidad o predisposiciones personales), además de influir en la percepción de Calidad de Vida de cada persona, afectan a las experiencias de aprendizaje, las que a su vez determinan y modifican las creencias de autoeficacia y expectativas de resultados ${ }^{17}$.

Actualmente, en Iberoamérica son pocas las investigaciones centradas en la Calidad de Vida en la niñez. En población no enferma, en Chile sólo se han encontrado referencias de estudios en población escolar y en la adolescencia 9 .

Es de vital importancia que más profesionales de la salud se involucren en el tema de Calidad de Vida infantil, ya que es suficientemente claro que las evaluaciones a temprana edad pueden ayudar a predecir aspectos del desarrollo y evolución durante la adolescencia y después de ella ${ }^{18}$.

Considerando entonces la influencia del contexto sociocultural en la evaluación que hacen los menores de su bienestar y que el estudio de la Calidad de Vida implica la evaluación tanto de factores del entorno material u objetivo como a factores psicosociales o subjetivos de los niños ${ }^{19}$, es que se hace necesario seguir desarrollando conocimientos en torno a la Calidad de Vida de este grupo etáreo, considerando las diferencias individuales, culturales y sociales, ya que estas son una base para comprender y lograr un mejor acercamiento a la realidad en que se desenvuelven.

En esta búsqueda de conocimientos en torno a la Calidad de Vida en niños se diseñó esta investigación que compara las visiones entregadas por los niños y niñas de la comuna urbana (Antofagasta) y una comuna rural (San Pedro de Atacama), dicha relevancia se da principalmente por el contraste de contextos en los cuales, nacen y crecen, principalmente la diferencia cultural que lleva a los niños el estar insertos en realidades distintas. Así el objetivo central del estudio es identificar los componentes significativos en la evaluación de la Calidad de Vida desde la percepción infantil.

A través de la investigación se busca dar respuesta a la pregunta ¿Cuáles son los dominios subjetivos relevantes y las dimensiones significativas en la evaluación de la Calidad de Vida, desde la percepción de los niños y niñas, en relación a dimensiones que según la teoría, constituyen la Calidad de Vida para los adultos?. Nuestra hipótesis pretende encontrar diferencias entre los menores pertenecientes a una zona rural y a una zona urbana.

\section{Participantes y Método}

Esta investigación se enmarca bajo un diseño no experimental de tipo transversal.

\section{Participantes}

El universo de estudio correspondió a la población de niños y niñas estudiantes de tercer año básico a sexto año básico de Establecimientos Educacionales Municipales de la Comuna de Antofagasta y de la Comuna de San Pedro de Atacama. El muestreo fue intencionado por disponibilidad.

Como criterios de inclusión se consideró: que los niños y niñas debían tener cumplidos 
los ocho años y no sobrepasar los doce años, saber leer y escribir y acceder voluntariamente a participar de la investigación. El criterio de inclusión de la edad se estableció a partir del instrumento.

Se realizaron dos mediciones: con el Cuestionario de Calidad de Vida Infantil y la entrevista con preguntas abiertas.

Fueron encuestados 200 participantes, 124 urbanos y 76 rurales. La tabla 1 muestra la distribución de la muestra por sexo y edad según localidad.

Las preguntas abiertas fueron contestadas por 192 sujetos, ya que ocho menores urbanos no asistieron al establecimiento cuando se realizó la segunda etapa. En el sector urbano participaron finalmente 116 menores, de los cuales el 50\% son mujeres. En el sector rural participaron 76 menores, de los cuales un $51,3 \%$ fueron mujeres.

\section{Instrumentos}

Escala de Calidad de Vida Infantil (CVI) ${ }^{20}$ : Instrumento autoadministrable que puede ser aplicable individual o colectivamente y que consta de 53 ítems politómicos, con 4 opciones de respuesta: "casi nunca", "pocas veces", "muchas veces" y "casi siempre". Fue construido en España por Sabeh, Verdugo y Contini en el año 2002. Evalúa CV infantil como una dimensión esencial e incluye las áreas de: bienestar emocional (BE), relaciones interpersonales (RI), desarrollo personal y actividades (DPA), bienestar físico (BF) y bienestar material $(\mathrm{BM})$.

Las características psicométricas de la escala CVI, fueron analizadas desde la Teoría de la Respuesta al Item (TRI), la fiabilidad de CVI, posee un índice de 0,98, y por lo tanto, altamente aceptable. En cuanto a la validez de contenido del cuestionario, la revisión teórica y de instrumentos, así como la consulta a jueces expertos que valoraron la adecuación de los ítems para evaluar los dominios

Tabla 1. Distribución de los participantes según sexo, edad y localidad

\begin{tabular}{|lccccc|}
\hline & $\begin{array}{c}\text { Comuna urbana } \\
\text { (n: 124) }\end{array}$ & \multicolumn{2}{c|}{$\begin{array}{c}\text { Comuna rural } \\
\text { (n: 76) }\end{array}$} \\
\hline $\mathrm{n}$ hombres (\%) & 61 & $(49,2 \%)$ & 36 & $(47,4 \%)$ \\
\hline $\mathrm{n}$ mujeres (\%) & 63 & $(50,8 \%)$ & 40 & $(52,6 \%)$ \\
\hline Media edad total (DE) & 10 & $(1,16)$ & 9,7 & $(1,20)$ \\
\hline Media edad hombres (DE) & 9,93 & $(1,20)$ & 9,75 & $(1,16)$ \\
\hline Media edad mujeres (DE) & 10,06 & $(1,13)$ & 9,65 & $(1,25)$ \\
\hline
\end{tabular}

definidos, dan apoyo a la misma. La validez de constructo se probó por el ajuste de datos al modelo, que fue altamente satisfactorio.

Esta escala ha sido aplicada también en Argentina y México.

Para efecto de comparar el peso de los distintos dominios y dada la desigualdad en el número de ítems que compone cada dimensión, los puntajes brutos fueron transformados a una escala de 0 a 100 puntos, donde a mayor puntaje, mejor $\mathrm{CV}$ reportada (tabla 2).

\section{Encuesta de preguntas abiertas}

Se aplicó una encuesta de tres preguntas abiertas, idénticas a las utilizadas por Verdugo y Sabeth en el 2002 y cuyo propósito general fue conocer las dimensiones de CV que con mayor frecuencia mencionan espontáneamente los niños cuando se les pregunta por sus experiencias de satisfacción, insatisfacción y deseos de cambio o mejora. Las preguntas fueron las siguientes siendo desarrolladas en forma escrita: 1. ¿Qué te puso muy contento, te hizo sentir feliz?; 2. ¿Qué te puso triste, pensativo o enojado? y 3 . ¿Qué te gustaría que cambie?

Las dimensiones utilizadas para clasificar las afirmaciones dadas por los niños fueron; ocio y actividades recreativas, rendimiento, relaciones interpersonales, bienestar físico/emocional, bienestar colectivo/valores y bienestar material.

\section{Procedimiento}

Previa autorización de los Directores de las Escuelas, se seleccionaron de forma no probabilística a los niños y niñas que participaron de la investigación y que cumplían con los criterios de inclusión.

A estos se les entregó la carta de consentimiento dirigida a los apoderados solicitando su autorización para la participación del/la menor. Esta carta debió ser firmada por el apoderado antes de la aplicación de los instrumentos de investigación. El asentimiento de los niños fue verbal. 
Tabla 2. Dominios evaluados en Calidad de Vida Infantil

\begin{tabular}{|c|c|}
\hline Dominio & Definición \\
\hline $\begin{array}{l}\text { Bienestar emocional } \\
\text { (13 Ítems) }\end{array}$ & $\begin{array}{l}\text { - Estados emocionales (afecto positivo/afecto negativo) experimentados por el niño como la alegría, } \\
\text { la tristeza, el nerviosismo, el humor, la preocupación } \\
\text { - Percepción global de satisfacción con la vida } \\
\text { - Visión de futuro (cómo cree que será su vida adulta) } \\
\text { - Autoestima/autoconcepto (cómo se ve y se valora a sí mismo y cómo cree que lo ven los demás en } \\
\text { general) }\end{array}$ \\
\hline $\begin{array}{l}\text { Relaciones interpersonales } \\
\text { (17 Ítems) }\end{array}$ & $\begin{array}{l}\text { - Frecuencia, calidad y satisfacción con afecto } \\
\text { - Interacción (positiva o negativa), comunicación y aceptación de y entre personas de la familia } \\
\text { - Estilo educativo y de crianza de los padres (premios, castigos, abandono) } \\
\text { - Frecuencia, calidad y satisfacción con las relaciones de amistad y compañerismo en el medio escolar } \\
\text { y extraescolar } \\
\text { - Satisfacción y calidad de las relaciones con los maestros; percepción por parte del niño de apoyos, } \\
\text { refuerzos, castigos, expectativas e imagen que tienen de sí los maestros }\end{array}$ \\
\hline $\begin{array}{l}\text { Desarrollo personal y } \\
\text { actividades } \\
\text { (11 Ítems) }\end{array}$ & $\begin{array}{l}\text { - Grado de desempeño, progreso, resultados y satisfacción personal con las actividades escolares de } \\
\text { aprendizaje } \\
\text { - Percepción que tiene el niño de sus habilidades cognitivas y oportunidades para desarrollarlas } \\
\text { - Frecuencia y oportunidad de experiencias de ocio y tiempo libre tales como: juegos, deportes, } \\
\text { actividad física, televisión, vídeos } \\
\text { - Autodeterminación (posibilidad de elegir y tomar decisiones) }\end{array}$ \\
\hline Bienestar físico & $\begin{array}{l}\text { - Estado de salud física del niño: sueño, enfermedades, síntomas y signos físicos, energía física, nivel } \\
\text { de nutrición, desarrollo físico (peso-altura) } \\
\text { - Acceso y satisfacción con la asistencia sanitaria }\end{array}$ \\
\hline Bienestar material & $\begin{array}{l}\text { - Posesiones materiales del niño y de la familia (regalos que recibe, ropa, juguetes, objetos) } \\
\text { - Características físicas de los ambientes en los que se desenvuelve (calidad y confort del hogar y del } \\
\text { - Nivel socioeconómico de la familia }\end{array}$ \\
\hline
\end{tabular}

La aplicación del instrumento se realizó en dos partes en los establecimientos escolares en las horas de clases y en forma grupal. Primero se les aplicó la escala de calidad de vida infantil, en la segunda etapa se encuestaron las tres preguntas abiertas que incluye el cuestionario las cuales fueron tomas en grupos pequeños.

Ambas actividades fueron desarrolladas por dos alumnas pertenecientes al equipo de la línea de investigación de Salud y Calidad de Vida de la UCN, quienes fueron capacitadas en la recolección de datos en menores.

Los datos fueron ingresados a una planilla en SPSS 15.0. Se realiza un análisis descriptivo de las variables. Para comprobar la hipótesis se aplicó una prueba $\mathrm{T}$ de student a fin de analizar la existencia de diferencias estadísticamente significativas entre las medias de los dominios de ambos grupos. El contenido de las preguntas abiertas fue analizado a través de frecuencia de respuesta.

\section{Resultados}

El dominio mejor evaluado en ambas localidades fue el del Bienestar Material (BM). Asimismo, el peor evaluado en ambos fue el del Bienestar Físico (BF). No se observan diferencias estadísticamente significativas en ninguna de las dimensiones evaluadas al comparar por tipo de localidad. Tampoco se encuentran diferencias estadísticamente significativas al comparar el puntaje entre hombres o entre mujeres en ambas localidades (tabla 3 ).

Al analizar la relación entre las variables de CV y edad, sólo es significativa la relación con Bienestar Emocional (BE) en los menores de la comuna urbana $(r=, 199 ; p<, 05)$ y con Bienestar Material (BM) en la comuna rural $(\mathrm{r}=, 269 ; \mathrm{p}<, 05)$. En ambas la correlación es directa, es decir, a medida que aumenta la edad, aumenta la CV percibida en dicha dimensión. 
Tabla 3. Medias y DE obtenidos por los menores en ambas localidades

\begin{tabular}{|c|c|c|c|c|c|c|c|}
\hline \multirow{2}{*}{\multicolumn{2}{|c|}{$\begin{array}{l}\text { Dominios de } \\
\text { CVI }\end{array}$}} & \multicolumn{3}{|c|}{ Comuna urbana } & \multicolumn{3}{|c|}{ Comuna rural } \\
\hline & & Hombres & Mujeres & Total & Hombres & Mujeres & Total \\
\hline \multirow[t]{3}{*}{ Bienestar emocional } & $\mathrm{n}$ & 53 & 59 & 112 & 32 & 34 & 66 \\
\hline & $x$ & 66,51 & 66,20 & $66,35^{*}$ & 64,50 & 65,70 & 65,12 \\
\hline & DE & 9,27 & 9,14 & 9,16 & 8,85 & 9,26 & 9,02 \\
\hline \multirow[t]{3}{*}{ Relaciones interpersonales } & $\mathrm{n}$ & 56 & 57 & 113 & 28 & 31 & 59 \\
\hline & $x$ & 61,02 & 58,14 & 59,57 & 58,54 & 59,52 & 59,05 \\
\hline & $\mathrm{DE}$ & 11,08 & 6,69 & 9,20 & 6,50 & 7,62 & 7,07 \\
\hline \multirow[t]{3}{*}{ Desarrollo personal y actividades } & $\mathrm{n}$ & 58 & 59 & 117 & 32 & 39 & 71 \\
\hline & $x$ & 67,43 & 65,11 & 66,27 & 63,90 & 61,89 & 62,80 \\
\hline & DE & 10,61 & 9,82 & 10,24 & 10,90 & 10,73 & 10,78 \\
\hline \multirow[t]{3}{*}{ Bienestar físico } & $n$ & 61 & 60 & 121 & 36 & 39 & 75 \\
\hline & $x$ & 54,98 & 56,51 & 55,74 & 54,14 & 56,31 & 55,27 \\
\hline & $\mathrm{DE}$ & 10,87 & 11,85 & 11,34 & 11,76 & 12,45 & 12,09 \\
\hline \multirow[t]{3}{*}{ Bienestar material } & $\mathrm{n}$ & 59 & 61 & 120 & 35 & 37 & 72 \\
\hline & $x$ & 74,03 & 77,43 & 75,76 & 74,17 & 73,03 & $73,58^{*}$ \\
\hline & DE & 13,47 & 12,37 & 12,98 & 14,25 & 12,10 & 13,10 \\
\hline \multirow[t]{3}{*}{ CV general } & $n$ & 47 & 50 & 97 & 21 & 25 & 46 \\
\hline & $x$ & 63,54 & 64,68 & 64,13 & 63,90 & 62,90 & 63,36 \\
\hline & $\mathrm{DE}$ & 6,73 & 5,92 & 6,32 & 8,01 & 7,42 & 7,62 \\
\hline
\end{tabular}

*La correlación es significativa al nivel ,05 (bilateral) con la edad.

Los resultados encontrados a través del análisis de contenido en cada una de las tres preguntas realizadas a los menores urbanos y rurales se clasificaron dentro de las seis dimensiones obteniendo porcentajes por frecuencia de respuestas.

La tabla 4 muestra los porcentajes de niños y niñas que expresan satisfacción en los dominios señalados por localidad. En el sector urbano, las mayores experiencias de satisfacción se dieron a nivel de las relaciones interpersonales, en tanto en el sector rural, el mayor porcentaje de satisfacción se da en torno a las actividades de ocio y actividades recreativas. Cabe mencionar el bajo porcentaje de menores que expresa satisfacción con su bienestar material en la comuna rural, lo que se refleja igualmente en el deseo de cambio en este dominio.

Es importante destacar asimismo que en ambos grupos las mayores experiencias de insatisfacción se dan a nivel de las relaciones interpersonales,
Tabla 4. Experiencias de satisfacción, insatisfacción y deseos de cambio en función de la localidad

\begin{tabular}{|llcc|}
\hline & Dimensión & $\begin{array}{c}\text { Comuna } \\
\text { urbana } \\
\%\end{array}$ & $\begin{array}{c}\text { Comuna } \\
\text { rural } \\
\%\end{array}$ \\
Experiencias de & Ocio y actividades recreativas & 26 & 51 \\
satisfacción & Relaciones interpersonales & 48 & 34 \\
& Bienestar material & 16 & 9 \\
Experiencias de & Relaciones interpersonales & 55 & 60 \\
insatisfacción & Bienestar físico y emocional & 22 & 29 \\
Deseos de & Relaciones interpersonales & 48 & 37 \\
cambio & Bienestar colectivo y valores & 24 & 17 \\
& Bienestar material & 13 & 34 \\
\hline
\end{tabular}

en tanto las de menos insatisfacción son en la esfera del Bienestar Físico y Emocional.

\section{Discusión}

A partir de este estudio se observó que utilizando pruebas objetivas comunes para ambos grupos de niños y niñas, no existirían diferencias en la evaluación de la CV entre los menores urbanos y rurales. Estos resultados en la percepción de los evaluados podrían ser explicados por el hecho de que los niños y niñas rurales 
han vivido un proceso de transformación e influencia extranjera, lo que en cierta medida los "homologaría" a los niños y niñas urbanas, en un proceso de interculturalidad significativo en sus vidas. Las dinámicas de aculturación pueden resultar en, a menudo difíciles, procesos constructivos de "creolización". Esto quiere decir que se producen zonas de contacto, $s p a-$ ces in between o "interfases entre sistemas" entre diferentes prácticas o ámbitos cultura$\operatorname{les}^{21}$. De estas zonas pueden surgir maneras interesantes de combinar diferentes estrategias de vida ${ }^{22}$, así también investigadores chilenos han encontrado diferencias estadísticamente significativas al comparar los niveles de resiliencia entre escolares urbanos y rurales, a favor de éstos últimos. Una posible explicación para lo anterior, tiene que ver con la estructura familiar preponderante en los sectores rurales, que tiende a ser más extensa, lo que se puede relacionar con la disponibilidad de redes y el tipo de contacto afectivo más cercano ${ }^{23}$.

En ambos grupos el dominio mejor evaluado es el de bienestar material y el peor evaluado el de bienestar físico.

Es necesario destacar que en el contenido aportado por las preguntas abiertas de los participantes de la comuna urbana, encontraron algunas diferencias, así, en la ciudad, las actividades que generan mayor grado de satisfacción en los menores evaluados correspondían al dominio de las relaciones interpersonales, a diferencia de los menores rurales en que pertenecían al dominio del ocio y las actividades recreativas. Posiblemente, las diferencias en el tipo de dominio que genera mayor satisfacción está vinculado al tipo de actividades predominantes en cada grupo, el cual puede estar mediatizado, probablemente, por el tiempo disponible, teniendo los menores rurales mayor posibilidad de realizar actividades recreativas y de ocio, dado que los establecimientos educacionales urbanos han privilegiado la asistencia de los menores en jornada completa, brindándoles menos oportunidades de realizar actividades de ocio y recreativas.

Paradójicamente, este mismo dominio que genera satisfacción en ambos grupos, es además el que causa mayor insatisfacción en ambas localidades, donde los menores plantean sus mayores deseos de cambio en frases tales como "Que mis papás no peleen" o "Que mis compañeros no me molesten". Cabe destacar que entre las actividades mencionadas la categoría de las relaciones interpersonales, se encuentran las interacciones con los amigos, familiares y conocidos, sumándose en el caso de niños rurales, los animales. Si consideramos el cuestionario objetivo, la salud y bienestar físico pareciera ser un dominio importante para este grupo de edad, independiente de la localidad, ya que fue el dominio peor evaluado en ambas mediante el cuestionario, pero contradictoriamente, fue el que genera menor insatisfacción, según las entrevistas. Esto puede ser explicado dado que al utilizar un cuestionario cerrado, el menor se ve forzado a evaluar aspectos específicos de su bienestar físico, sin embargo, al preguntar abiertamente por actividades que generan insatisfacción, este no se constituye como un elemento importante en la vida cotidiana de los menores, dado a que en general a esa edad presentan una buena salud y se encuentran en un buen estado físico, aportando escasamente a los factores importantes para los menores al evaluar su $\mathrm{CV}$.

En relación a lo anterior, se observan estudios que por un lado apuntan a que los niveles de salud son más bajos en niños de zonas rurales ${ }^{24,25}$ y por el contrario, se sabe también que muchos niños de zonas urbanas viven en extrema pobreza, con sus derechos negados, sin tener sus necesidades cubiertas y con un provenir perjudicado por factores que amenazan su salud y mina su desarrollo ${ }^{26}$.

Aun cuando en el cuestionario ambos grupos mencionan como el dominio mejor evaluado el de bienestar material, las entrevistas a los menores reflejan que en el sector rural este dominio sólo sería fuente de satisfacción en menos de un $10 \%$ de los evaluados, siendo relevante para este grupo de menores, quienes incluso lo mencionan como una de las principales fuentes de satisfacción ante posibles cambios.

\section{Conclusiones}

Los resultados del estudio muestran la inexistencia de diferencias significativas en la 
percepción de $\mathrm{CV}$ entre menores urbanos y rurales a partir del cuestionario, sin embargo, el contenido obtenido en las preguntas abiertas, permitió establecer algunas diferencias entre los grupos.

Las diferencias entre ambos grupos, las que no pudieron ser detectadas a través del cuestionario genérico, permiten aventurar la necesidad de utilizar instrumentos que sean capaces de recoger información relevante y contextualizada al grupo que estamos evaluando, ya que sólo así seremos capaces de detectar los reales factores involucrados en la evaluación del bienestar y la CV de los menores, considerando que, tal como lo planteado por Quiceno y Vinaccia ${ }^{27}$, la CV infantil sería la percepción del bienestar físico, psicológico y social del niño o adolescente dentro de un contexto cultural específico de acuerdo a su desarrollo evolutivo y a sus diferencias individuales.

Posiblemente los elementos o factores sean similares, sin embargo, la significación que se le otorga a cada componente variará dependiendo del grupo, lo que amerita la necesidad de al momento de utilizar cuestionarios estandarizados, considerar la importancia que le atribuye cada uno de los sujetos a la dimensión evaluada, ya que ésta variará de acuerdo a su momento evolutivo, su historia de vida y su contexto socio-cultural.

Las relaciones interpersonales presentan un peso importante en la percepción de satisfacción en la muestra total de evaluados.

Una limitación de este estudio tiene relación con la muestra, debido que al ser intencionada por disponibilidad, no se tienen garantías que los datos sean representativos de la población por lo que la inferencia estadística debe ser tomada con cautela.

Futuras investigaciones deberían incorporar una medición del grado de aculturación en los menores, con el objetivo de observar la influencia de los contextos socioculturales.

\section{Referencias}

1.- WHOQoL GROUP: The World Health Organization Quality of Life Assessment (WHOQOL). Position Paper from the World Health Organization. Soc Sci Med 1995; 41: 1403-9.
2.- Urzúa A, Caqueo-Urizar A: Calidad de Vida, una revisión teórica del concepto. Terapia Psicológica 2012; 30 (1): 61-71.

3.- Urzúa A: CV relacionada con la salud: elementos conceptuales. Rev Med Chile 2010; 138: 358-65.

4.- Rajmil L, Roizen M, Urzúa A: CV y salud en la infancia y la adolescencia. Revista Típica 2010; 6 (2): 244-9.

5.- Moreno B, Kern E: CV relacionada con la salud infantil y el trasplante de órganos: una revisión de la literatura. Revista Colombiana de Psicología 2005; 14: 46-52.

6.- Gómez-Vela M, Sabeh E: CV. Evolución del concepto y su influencia en la investigación y la práctica. Integra 2005; 3 (9): 1-4.

7.- Urzúa A, Mercado G: La evaluación de la CV de los y las adolescentes a través del KIDDO- KINDL. Revista Terapia Psicológica 2008; 26 (1): 133-41.

8.- Urzúa A, Cortés E, Prieto L, Vega S: Autoreporte de la CV en niños y adolescentes escolarizados. Rev Chil Pediatr 2009; 80 (3): 238-44.

9.- Urzúa A, Méndez F, Acuña C, Astudillo J: CV relacionada con la salud en edad preescolar. Rev Chil Pediatr 2010; 81 (2): 128-37.

10.- Urzúa A, Avendaño F, Díaz S, Checura D: CV y conductas alimentarias en La preadolescencia. Rev Chil Nutr 2010; 37 (3): 282-92.

11.- Cadiz V, Urzúa A, Campbell M: CV en niños y adolescentes sobrevivientes de leucemia linfoblástica aguda. Rev. Chil. Pediatr 2011; 82 (2): 113-21.

12.- UNICEF/Centro de investigaciones Innocenti: Innocenti Insight: La evolución de las facultades del niño. Estocolmo: Gerison Lansdown: 2000. Extraído el 10 de Junio del 2006 de http://www.unicef.cl/derecho/ antecedentes.htm.

13.- Apud A: Participación Infantil. Enrédate con UNICEF: 2000. Extraído el 01 de Diciembre 2011 de http://www. sename.cl/wsename/otros/unicef.pdf.

14.- Verdugo M, Sabeh E: Evaluación de la percepción de CV en la infancia. Psicothema 2002; 14 (1): 86-91.

15.- Verdugo M: El cambio educativo desde una perspectiva de CV. Revista de Educación 2009; 349: 23-43.

16.- Maita M, Peralta O: El impacto de la instrucción en la comprensión temprana de un mapa como objeto simbólico. Infancia y Aprendizaje 2010; 33 (1): 47-62.

17.- Cupani M, Lorenzo J: Evaluación de un modelo social-cognitivo del rendimiento en matemática en una población de preadolescentes argentinos. Infancia y Aprendizaje 2010; 33 (1): 63-74.

18.- Margarita J, Vinaccia $S$ : CV relacionada con la salud infantil: una aproximación conceptual. Psicología y Salud 2008; 18 (001): 37-44. 
19.- Salas $L$ : "CV" y "la nueva sociología de la infancia", perspectivas de investigación social complementarias. Hologramática 2012; 12 (4): 83-95.

20.- Sabeh E, Verdugo M, Contini E: CVI-CVIP. Cuestionarios de evaluación de la CV en la infancia: 2002. Madrid: CEPE.

21.- Temple D, Layme F, Michaux J, Gonzales M, Blanco E: Las Estructuras Elementales de la Reciprocidad: 2003. TARI, Plural Editores. UMSA, La Paz.

22.- De Munter K: Nayra, Ojos al Sur del Presente. Aproximaciones antropológicas a la interculturalidad contemporánea: 2007. Latinas Editores-CEPA, Oruro.

23.- Castro A, Saavedra E, Saavedra P: Niños de familias rurales y urbanas y desarrollo de la resiliencia. Revista
Iberoamericana De Psicología: Ciencia y Tecnología 2010; 3 (1): 109-19.

24.- Cherry D, Huggins B, Gilmore K: Salud de los niños en el ambiente rural. Pediatr Clin N Am 2007; 54: 121-33.

25.- González M, Castillo C: Crecimiento postnatal inicial de lactantes prematuros nacidos en Chillán, de acuerdo a residencia urbana o rural. Rev Chil Pediatr 2001; 72 (6): 496-503.

26.- UNICEF, Centro de Investigaciones Innocenti: Pobreza y exclusión entre niños Urbanos. Digest Innocenti 2002, 10.

27.- Quiceno J, Vinaccia M: CV relacionada con la salud infantil, una aproximación conceptual. Psicología y Salud 2008; 18 (1): 37-44. 